\title{
CONFLICTOS SOCIALES Y PUEBLOS INDÍGENAS: DEFENSA DEL DERECHO FUNDAMENTAL AL MEDIO AMBIENTE
}

\author{
SASACHAKUYKUNA HINALLATAQ INDIGENA \\ LLAQTAKUNAWAN: MAMAPACHAQ DERECHO NISQA \\ SAYAPAKUQ
}

\section{SOCIAL CONFLICTS AND INDIGENOUS PEOPLES: DEFENSE OF THE FUNDAMENTAL RIGHT TO THE ENVIRONMENT}

\section{Milagros Chaska Alvarado Yucra ${ }^{(1)}$}

Ch'uyanchakuy: Kay qillqa rurasqaypi riqsichisaq imaymana sasachakuykunapi, chiqninakuykunapi mama llaqtanchikuna Estado nisqawan kawsasqanmanta, imaraykuchus chay Tayta Estado allpanchikkunamanta, pachamamanchikkunamanta imaymana kawsay waqaychasqantapas urqunankupaq haywayapusqanmanta, chayraykutaqmi pachanchik qillichasqa ñakarichkan,imaymananchikpurmachkan, chaymiderechosnisqakunasarunchasqa tarikun, achwanpas pachamamanchikpa derecho chaninchasqan, chay 22 yupaypi, 2 artículo, Constitución Política del Perú nisqapa riqsisqan. Kaykunapa huchanpin mama llaqtakunaqa Estadupa políticas churasqanpi mañana iñinkuchu, chay Ley de Consulta Previa nisqapi hina, iskay uya tuqlla política nisqapi kutirpapuspa, chay qullqiyuq empresa nisqakunaman sayaparpakun, chayraykupin llaqtanchikkunaqa unpuchasqapuni qipanku, chay empresas extractiva nisqawan mana imata rurayta atispa. Kayna kachkaptin, Estaduqa Ilullarikuspa qullqiyuqkunata apamusun qullqinkuwan yanapawasun nispa, mana imatapas llaqtanchikkunapa derecho nisqan amachananpaqqa, qullqiyuqkunaman sayapakuspa ruranchu. Ichaqa chay Constitución nisqapiqa kachkanmi kamachisqa mecanismos procesales nisqa, garantías constitucionales nisqawan sutichasqata, chiqa derechunchikkuna amachananpaq.

Yuyaysapa simikuna: Pachamamaman dirichuyuq kanchik, Ñawpaqta tapukuy, Mama llaqtakuna, Qawanakuykuna, Allin kamachiykuna. 
Resumen: Este artículo trata de los conflictos sociales entre los Pueblos Indígenas y el Estado, originados a causa de las concesiones otorgadas por el Estado para la explotación de los recursos naturales en los territorios indígenas. Esto genera contaminación ambiental y destrucción de ecosistemas, la vulneración de varios derechos, entre ellos el derecho al medio ambiente (Art. 2, 22 Constitución Política del Perú). Los Pueblos Indígenas desconfían de las políticas públicas y el Derecho como es en el caso de la Ley de Consulta Previa, percibida como una suerte de aditamento a favor de las empresas extractivas. Frente a esta situación, con la excusa de promover la inversión privada, el Estado ha hecho poco por defender los derechos indígenas. Por otro lado, la Constitución establece garantías constitucionales.

Palabras claves: Derecho al medio ambiente, Consulta previa, Pueblos indígenas, Conflictos sociales, Garantías constitucionales.

Abstract: This article deals with the social conflicts between Indigenous Peoples and the State, originated on the concessions granted by the State for the exploitation of the natural resources in the indigenous territories. This generates environmental pollution and destruction of ecosystems, the violation of several rights, including the right to the environment (Art. 2, 22 Political Constitution of Peru). Indigenous Peoples distrust public policies and Law as it is in the case of the Law of Prior Consultation, perceived as a sort of addition in favor of extractive companies. Faced with this situation, with the excuse of promoting private investment, the State has done little to defend indigenous rights. On the other hand, the Constitution establishes constitutional guarantees.

Key words: Environmental rights, Previous consultation process, Indigenous peoples, Social conflicts, Constitutional guaranties. 


\section{Introducción}

El escenario de la economía global fue reconfigurado a causa de los diversos hechos históricos de envergadura, generando un proceso de auge y expansión de las actividades económicas, siendo el sector más beneficiado, el de las industrias extractivas. Particularmente, en América Latina, a partir de los años noventa, las inversiones por parte de empresas extractivas fueron aumentando, debido a su promoción por parte de los Estados.

En el caso del Perú, se ha "promovido una serie de reformas con el objetivo de convertir al país en un destino atractivo para la inversión privada y consolidar una economía receptiva del capital, en especial extranjero" (ALAYZA MONCLOA 2007, 18). Sin embargo, dichas reformas se realizaron y se siguen realizando, sin tomar en cuenta los efectos negativos que traen las actividades extractivas en las zonas donde se ejecutan, puesto que se vulneran varios derechos fundamentales, como es el derecho al medio ambiente, particularmente de los pueblos indígenas, hecho que ha generado varios conflictos sociales.

Sin embargo, la Constitución Política del Perú, a través de las garantías constitucionales, proporciona mecanismos para defender los derechos fundamentales que se vulneran a causa de la explotación de nuestros recursos naturales, los que son poco usados en estos casos.

Por lo que es importante conocer qué son los derechos fundamentales para, de este modo, abordar en específico la importancia del derecho fundamental al medio ambiente y su reconocimiento en nuestra legislación. Ello nos permitirá entender que efectos negativos trae la contaminación y destrucción de ecosistemas a causa de las actividades extractivas. La mayoría de las actividades extractivas se desarrollan en territorios de los Pueblos Indígenas, que son directamente afectados por los efectos negativos de las actividades extractivas; son desplazados de dichas zonas, adquieren enfermedades a causa de los elevados índices de contaminación, entre otras situaciones, que han generado diversos conflictos sociales por la defensa de sus derechos.

\section{2. ¿Qué entendemos por Derechos Humanos y Derechos Fundamentales?}

Antes de hablar del tema central, es necesario conocer qué son los derechos humanos y los derechos fundamentales, para poder entender la importancia de la defensa del derecho fundamental al medio ambiente en caso de conflictos sociales entre los Pueblos Indígenas y el Estado o las empresas extractivas.

Al respecto cabe indicar que los derechos humanos han ido evolucionando a lo largo de la historia, sin embargo no han perdido su esencia inicial, pues se 
configuran como aquellas exigencias universales derivadas de la dignidad humana. Tienen su sustento en diversas teorías, como las teorías iusnaturalistas que concibe a los derechos humanos como aquellas facultades inherentes a la naturaleza humana, que le corresponden a todo ser humano, por el mismo hecho de serlo.

Conforme a la teoría historicista, los derechos humanos han pasado por diversas etapas, por lo que su reconocimiento es de género histórico; en cambio, la teoría pragmática centra el fundamento de los derechos fundamentales en la necesidad de su protección. La teoría contractualista, basada en el contrato social, cuyo sustento indica que el ejercicio de nuestros derechos no puede perjudicar a los derechos de los demás, por lo que debemos actuar conforme a los lineamientos estatales supeditados en el contrato social. Finalmente, la teoría positivista, dice que los derechos humanos encuentran su fundamento en su reconocimiento en un ordenamiento normativo, por lo que el componente jurídico normativo adquiere especial relevancia.

Sin embargo, pese a la existencia de estos sustentos, la posición ius naturalista es la que prevalece sobre las demás teorías. A partir de ello y considerando los diversos hitos históricos, se conceptúa a los derechos humanos como "demandas de abstención o actuación, derivadas de la dignidad de la persona y reconocidas por la comunidad internacional, siendo por ello merecedoras de protección jurídica por el Estado" (ESCOBAR ROCA 2005, 16), es decir, los derechos humanos son el conjunto de facultades e instituciones que en cada momento histórico, concretan exigencias de la dignidad, la libertad y la igualdad humana, las cuales deben ser reconocidas positivamente por los ordenamientos jurídicos a nivel nacional e internacional.

Es por ello que, ante la necesidad de garantizar el cumplimiento de los derechos humanos, los Estados modernos dan origen a cuerpos normativos que constituyen la norma suprema de los Estados, nos referimos a las Constituciones. Así, se dan los primeros procesos de constitucionalización de derechos, donde se incorporan los derechos humanos a las Constituciones, los derechos humanos positivizados adquieren la denominación de derechos fundamentales y dicha incorporación al texto constitucional implica un nivel muy elevado de protección. Cabe precisar que no basta que los derechos fundamentales sean reconocidos en la Constitución, sino es necesario que la Constitución sea reconocida en la legislación como el cuerpo normativo de mayor jerarquía normativa. Es decir, los derechos fundamentales "son aquellos derechos que el poder constituyente, máxima expresión jurídica de la soberanía popular, ha considerado los más importantes, los seleccionados para gozar del mayor nivel de garantía" (ESCOBAR ROCA 2005, 27).

Posteriormente, se da el proceso de Internacionalización, que surge al crearse la ONU, después de la Segunda Guerra Mundial, se comienza a hablar de las 
obligaciones internacionales de los Estados, que implica que los derechos humanos no solo sean reconocidos en las Constituciones, sino que estos se reconocen en Tratados, que son acuerdos internacionales por parte de los Estados, por lo que se habla del Derecho Internacional de los derechos humanos, y se empieza a reconocer los derechos en los tratados incorporándolos en las Constituciones. Es un proceso que aún no concluye, ya que a causa de la globalización y otros factores van apareciendo más derechos, que aún no están incorporados en las Constituciones y también hay derechos en las Constituciones que no aparecen en tratados.

Entonces, "los derechos humanos son entendidos básicamente como demandas derivadas de la dignidad de la persona, pertenecen a un campo de la Ética, no del Derecho positivo" (ESCOBAR ROCA 2005, 33). Los mismos que al ser reconocidos por una Constitución vigente y concreta, se transforman en derechos fundamentales, y dejan de ser sólo demandas, para convertirse en exigencias jurídicamente tuteladas.

\section{El derecho fundamental al medio ambiente y su importancia}

Teniendo claro qué son los derechos humanos y los derechos fundamentales, pasaremos a desarrollar el derecho fundamental al medio ambiente, sin embargo, antes es necesario hacer mención a las tres categorías en las cuales se clasifican los diferentes derechos, para poder identificar en cuál de estas categorías se encuentra este derecho. Según la clasificación tradicional, los derechos se han clasificado en derechos de primera generación o derechos civiles y políticos, que son los derechos inherentes a la calidad de ser humano, como el respecto a la libertad e igualdad. Los derechos de segunda generación o los derechos económicos sociales y culturales, que son aquellos que garantizan que todas las personas puedan acceder a condiciones mínimas que posibiliten su vida digna en sociedad, como el derecho a la vivienda, a la educación, entre otros. Asimismo, se tiene a los derechos de tercera generación o derechos colectivos, donde la sociedad es la titular, que buscan proteger y preservar al colectivo, dentro de éstos encontramos el derecho al medio ambiente, el derecho a la paz, entre otros.

Respecto al derecho al medio ambiente, existen diversos instrumentos internacionales que lo reconocen. Se hace mención de este derecho por primera vez en la Declaración de Estocolmo sobre Medio Humano. Asimismo, la Declaración Universal de Derechos Humanos refiere que "Toda persona tiene el derecho a un nivel de vida adecuado que le asegure, así como a su familia, la salud y el bienestar (...)". Del mismo modo, se hace referencia a este derecho en el Pacto Internacional de Derechos Económicos, Sociales y Culturales, la Declaración de las Naciones Unidas sobre el Medio Ambiente, la Cumbre de Río, la Cumbre de Johannesburgo, entre otros. 
Estos instrumentos internaciones, se configuran como "mecanismos de índole internacional y nacional en protección del medio ambiente y los recursos naturales, así como políticas públicas por parte de los Estados tendientes a proteger estos derechos que pertenecen a toda la humanidad" (VIDAL RAMOS 2013, 32). Debido a la importancia que comienza a tener este derecho, los Estados comienzan a incorporarlo y reconocerlo en sus constituciones, para darle un mayor nivel de protección.

Al respecto, tenemos que en nuestro ordenamiento jurídico, la Constitución de 1979, reconoce el derecho a un ambiente saludable y equilibrado, el mismo que es incorporado en el Capítulo referido a los Recursos Naturales, ubicado en el Título sobre el Régimen Económico, que establecía:

Artículo $123^{\circ}$ : Todos tienen el derecho de habitar en ambiente saludable, ecológicamente equilibrado $y$ adecuado para el desarrollo de la vida y la preservación del paisaje y la naturaleza. Todos tienen el deber de conservar dicho ambiente. Es obligación del Estado prevenir y controlar la contaminación ambiental.

Del mismo modo, la Constitución de 1993, reconoce este derecho en el Capítulo I (Derechos Fundamentales de la persona), del Título I (De la persona y de la sociedad), que refiere:

Artículo 2. Toda persona tiene derecho: 22. A la paz, a la tranquilidad, al disfrute del tiempo libre y al descanso, así como a gozar de un ambiente equilibrado y adecuado al desarrollo de su vida.

Al respecto, cabe indicar que, la Constitución de 1979, a través del artículo 123, reconocía un derecho y una obligación de alcance general para todas las personas y establecía que era una obligación del Estado prevenir y controlar la contaminación ambiental. Sin embargo, nuestra Constitución actual, en el numeral 22 del artículo 2, no refiere que el Estado este obligado a preservar el medio ambiente o que evite la contaminación ambiental. Estos aspectos, se establecen en el Capítulo II (Del Ambiente y los Recursos Naturales) del Título III (del Régimen económico), en los artículos 67, 68 y 69 de la Constitución, en base a los cuales, el Estado determina la política ambiental a nivel nacional, regional y local, a través del Poder Legislativo, Ejecutivo y Judicial. Mediante los artículos antes mencionados, "el Estado está obligado a promover: El uso sostenible de los recursos naturales, la conservación de la diversidad biológica, las áreas naturales protegidas, y el desarrollo de la Amazonía con una legislación adecuada" (VIDAL RAMOS 2013, 36).

Al respecto, el Tribunal Constitucional ha indicado que al hablar de un ambiente equilibrado, hacemos referencia a: (...) el conjunto de bases naturales de 
la vida y su calidad, lo que comprende, a su vez, los componentes bióticos, como la flora y la fauna, y los abióticos, como el agua, el aire o el subsuelo, los ecosistemas e, incluso, la ecósfera, esto es, la suma de todos los ecosistemas, que son las comunidades de especies que forman una red de interacciones de orden biológico, físico y químico (Caso Alida Cortez Gómez de Nano. 2002, 12).

En ese sentido, la importancia del derecho al medio ambiente, radica en que gracias al real goce y disfrute de éste, se pueden materializar otros derechos constitucionales, como el derecho a la vida, a la salud, a la vivienda, entre otros. Motivo por el cual, la positivización del derecho al medio ambiente es un paso fundamental para la sociedad, ya que refleja la toma de conciencia ante la problemática ambiental que se vive actualmente, sin embargo, ello no debe ser una mera declaración formal.

\section{Los pueblos indígenas y la consulta previa}

El Estado peruano ha reconocido de forma oficial que las comunidades campesinas y nativas constituyen pueblos indígenas. Así lo informo el Estado a la Comisión de Expertos en Aplicación de Convenios y Recomendaciones de la OIT (RUIZ MOLLEDA 2013, 115). Es así que, conforme el artículo 1 del Convenio Colectivo 169 de la OIT, para que los pueblos indígenas sean reconocidos como tal, deben descender de pueblos originarios y conservar total o parcialmente sus instituciones, así como poseer la autoconciencia de su identidad indígena o tribal.

Estos grupos, se han configurado como actores de resistencia frente a la explotación indiscriminada de los recursos naturales, debido a que dichas poblaciones resultan directamente impactadas por la contaminación generada a causa de las actividades extractivas, que implica la vulneración de sus derechos. Hecho que ha generado diversos conflictos sociales, debido a que el Estado dispone los territorios de dichas poblaciones de manera arbitraria, superponiendo proyectos extractivos y de infraestructura, sin consulta y con limitados mecanismos de participación, generando impactos sociales y ambientales muy serios, reduciendo estándares y capacidades de fiscalización ambiental, con la excusa de promover las actividades extractivas y generar el bienestar nacional, a partir de los ingresos que estas actividades generaban al Estado, sin tomar en consideración los derechos fundamentales de los pueblos indígenas.

Es así que, ante estos conflictos sociales, particularmente el conflicto de Bagua en el año 2009, y con la finalidad de lograr conciliar las visiones y aspiraciones de los pueblos indígenas con las necesidades de las mayorías nacionales, se promulgó la Ley de Consulta Previa, Ley № 29785, en septiembre del 2011; y fue reglamentada en abril del 2012. El primer proceso oficial de consulta realizado en virtud de esta ley se inició en mayo de 2013 en el caso Maijuna-Kichwa. 
Sin embargo, esta ley no ha traído consigo avances significativos, debido a que resulta ser una forma de "trampa política", porque no tiene eficacia para proteger los derechos colectivos de los pueblos indígenas, en razón a que Estado y las empresas extractivas han utilizado diversos argumentos y pretextos jurídicos, para incumplir lo estipulado en la Ley de la Consulta previa. Las causas que hacen que la consulta previa no funcione en el Perú son (RUIZ MOLLEDA 2015):

1) No está claro cuál es el territorio de los pueblos indígenas, lo que genera inseguridad jurídica respecto a sus territorios y posibilita que el Estado pueda entregar todo tipo de derechos sobre estos territorios.

2) Mediante la Directiva № 03-2012/MC, para ser considerado pueblo indígena y aplicar el derecho a la consulta, se exige permanecer en el territorio o conservar la lengua, esta Directiva se ha constituido en un mecanismo para evadir el proceso de consulta previa.

3) La segunda disposición final de la Ley de consulta previa, establece que no modifica ni deroga medidas legislativas y administrativas anteriores a la fecha de publicación de esta norma, con el cual convalida y blinda los actos administrativos y normativos expedidos entre el 1995 y el 2011.

4) Las empresas siguen realizando talleres informativos en vez de consulta, a pesar de que la Corte Suprema y el Tribunal Constitucional han señalado que los talleres informativos no exoneran al Estado de su obligación de realizar la consulta.

5) Mediante el acuerdo previo, se viene evadiendo el proceso de la consulta, en condiciones de asimetría de poder, sin la presencia del Estado, que implican la renuncia de derechos.

6) En las concesiones mineras se expiden en territorios de pueblos indígenas a espaldas de ellos, desprotegiendo sus derechos, a pesar del enorme impacto que generan las concesiones mineras en sus territorios.

7) El procedimiento para pedir consulta previa es absolutamente excluyente, ya que los pueblos indígenas nunca se enteran de la emisión de concesiones mineras porque no se les notifica.

8) No se consulta las concesiones mineras. No obstante que la concesión minera es la primera decisión que afecta el derecho de propiedad sobre el territorio de comunidades campesinas y nativas, el Ministerio de Cultura, el Ministerio de Energía y Minas e INGEMMET insisten en no consultar esta primera decisión.

Se tiene que, hasta marzo de 2017 son 28 los procesos de consulta previa terminados: 11 en hidrocarburos, 8 en minería, 4 Áreas Naturales Protegidas. Sin embargo, los procesos de consulta previa realizados, no cumplen los estándares que establece el Convenio 169 de la OIT, debido a que los actos administrativos consultados no son idóneos y relevantes para el caso concreto, ya que la 
autoridad sólo se remite a verificar que los requisitos exigidos al solicitante estén completos. Asimismo, en el proceso de consulta existe una asimetría informativa, puesto que los pueblos indígenas están ante una clara desventaja, debido a que las empresas extractivas, no brindan la información específica en torno a los impactos de exploración y explotación extractiva en los derechos colectivos de los pueblos indígenas, es decir, manejan la información a su favor. Por lo que, los acuerdos que se realizan son bastante generales y de mera formalidad, con la intención de justificar el cumplimiento de las obligaciones del Estado y hacer dar a conocer los compromisos y obligaciones de la empresa extractiva. "No basta realizar una consulta previa adecuada, sino que es necesario que se promulgue la medida a consultar y comprobar así si los acuerdos llegados son adecuados o no" (COORDINADORA NACIONAL DE DERECHOS HUMANOS 2017, 11).

Como sabemos, la vulneración de los derechos de los pueblos indígenas, a causa de las actividades extractivas, no sólo se da en nuestro país, frente a esta situación, la propuesta indígena andina es la siguiente:

"Como hijos de la Madre Tierra, los pueblos indígenas andinos tenemos una opción distinta, sustentada en el buen vivir, en la armonía y el diálogo con la naturaleza. Y en las dos últimas décadas, hemos avanzado sustancialmente en nuestra organización y articulación. Nuestro reto es hacer realidad el pleno ejercicio de nuestros derechos conquistados, todo ello compartiendo y construyendo con el conjunto de movimientos sociales una alternativa distinta al extractivismo capitalista neoliberal" (PALACÍN QUISPE 2017).

\section{Conflictos sociales por la defensa del derecho al medio ambiente}

Frente a los diversos casos de contaminación ambiental suscitados a consecuencia de las actividades extractivas, se han generado diversos conflictos sociales, particularmente entre el Estado y los pueblos indígenas. En dicho contexto, el Estado estableció políticas a favor de las actividades extractivas, sin considerar los derechos fundamentales que se vulneraban.

Es así que, en el gobierno de Alberto Fujimori, se dieron diversos cambios estructurales para promover la inversión privada, que implicó la regresión en la protección de derechos humanos y medioambientales, particularmente los derechos de propiedad colectiva de pueblos y comunidades indígenas. En el gobierno de Alejandro Toledo, se consolidaron las reformas hechas por Fujimori, además se aprobó la Ley 28259 (Ley de reversión a favor del Estado de los predios rústicos adjudicados a título gratuito). Del mismo modo, en el gobierno 
de Alan García se implementaron más políticas a favor de la inversión privada, a través de los Tratados de Libre Comercio (TLC), los Decretos Legislativos 1015 y el 1073 (referidos a la flexibilización de la toma de decisiones en las comunidades campesinas de la sierra y comunidades nativas con relación a actos de disposición de la propiedad colectiva), los mismos que fueron derogados ante las diversas protestas de la población amazónica, siendo el más resaltante el conflicto conocido como el "Baguazo", ocurrido el 5 de junio de 2009, donde indígenas de la Amazonía se enfrentaron con efectivos policiales, teniendo como resultado la muerte de varias personas. Motivo por el cual, en el gobierno de Ollanta Humala, con la finalidad de que conflictos, como los sucedidos en Bagua, no se vuelvan a repetir, se promulgó la Ley 29785 - Ley del Derecho a la Consulta Previa a los Pueblos Indígenas u Originarios, reconocida en el Convenio 169 de la Organización Internacional del Trabajo, que de una u otra manera, respaldaba los derechos de los pueblos indígenas en el Perú, aunque en la práctica, no ha logrado el cese o disminución de los conflictos sociales.

En diciembre de 2017, según el Reporte de Conflictos Sociales Nº16 de la DEFENSORÍA DEL PUEBLO (DEFENSORÍA DEL PUEBLO 2017), se da cuenta de 169 conflictos sociales: 119 activos y 50 latentes, de los cuales 120 (71\% del total) son de tipo socioambiental, que comprenden las siguientes actividades: minera (78), hidrocarburos (17), energía (8), otros (7), residuos y saneamiento (5), agroindustrial (3) y forestales (2). En este escenario de expansión, la disputa por el control de recursos escasos, como es el caso de las tierras agrícolas, los recursos hídricos, los temas de contaminación, desplazamiento de poblaciones y actividades productivas, son algunos de los aspectos centrales que están en la base de los conflictos entre las empresas extractivas y comunidades campesinas y nativas"(DE ECHAVE CÁCERES 2012, 15).

El incremento de los conflictos sociales en los últimos años, se ha dado en razón a diversos factores. A continuación, detallamos algunos de los más importantes (DE ECHAVE CÁCERES 2012, 29):

1. Un marco legal que ha brindado condiciones extremadamente favorables a las empresas extractivas.

2. Mientras que la minería y los hidrocarburos crecían en importancia y eran priorizados como actividades estratégicas en el Perú, los derechos de las poblaciones rurales, sobre todo comunidades campesinas e indígenas, eran claramente afectados.

3. Un marco de normas ambientales débil, caracterizado por la ausencia de un enfoque transectorial, la falta de institucionalidad y recursos para liderar la gestión ambiental, las insuficientes normas referidas a los limites máximos permisibles de contaminación, los pasivos ambientales irresueltos.

4. Los escasos mecanismos de participación ciudadana y consulta. 
Teniendo en cuenta que las actividades extractivas están presentes en casi todo el país, debemos hacer referencia de que dicha actividad siempre genera contaminación ambiental, dando origen a diversos casos de contaminación en perjuicio de la población y el patrimonio ambiental, que ha generado diversos conflictos sociales, siendo los más relevantes: la contaminación por mercurio en Choropampa generada por la Minera Yanacocha (el mismo que finalizó en un proceso judicial que devino en el Primer Pleno Casatorio de la Corte Suprema de la República del Perú), la consulta del Lote 192, tras los graves impactos dejados tras 40 años de explotación petrolera irresponsable (región de Loreto), la omisión de consulta de Lote 116 (región de Amazonas), los derrames de petróleo en ríos amazónicos (región de Loreto), el proceso de consulta previa del proyecto de Hidrovía Amazónica (región de Loreto), la omisión de pago de servidumbres petroleras (región de Loreto), la omisión de titulación de territorios de pueblos indígenas (regiones de Amazonas, Loreto, Ucayali y Junín), la ejecución de actividades extractivas en territorios de pueblos indígenas en aislamiento voluntario y contacto inicial en el Lote 88 (regiones de Cusco, Ucayali y Madre de Dios), las denuncias contra el gobierno por su inactividad ante la contaminación en Espinar (región de Cusco), la omisión de consulta de construcción de línea de trasmisión de electricidad Moyobamba (región de lquitos), la actividad minera en cabecera de ríos del Cenepa (región de Amazonas), el proceso del Baguazo o el derecho a la protesta (región de Amazonas), la omisión de consulta de concesiones mineras (región de Puno), entre otros.

Los conflictos antes mencionados, ocurridos a causa de problemática ambiental, son una clara evidencia de que es necesario que Estado peruano implemente políticas públicas que garanticen el real goce del derecho al medio ambiente, asimismo que logren prevenir, proteger y restaurar de manera real y efectiva este derecho, puesto que en la actualidad se habla con mayor énfasis de procesos de desarrollo basados en la diversidad de los recursos y la promoción del uso sostenible de los recursos.

\section{Tutela procesal del derecho al medio ambiente}

Dentro de este contexto, se han establecido diversas formas de proteger el derecho fundamental medio ambiente, a través de los procesos constitucionales establecidos en nuestra Constitución, acciones civiles referidas a la reparación civil por daño ambiental, acciones penales ante la comisión de delitos ambientales, la Ley del Proceso Contencioso-Administrativo y la Ley General del Ambiente N. ${ }^{\circ}$ 28611. Sin embargo, no se ha identificado cuál de estos procedimientos es el más eficaz y apropiado para la protección del derecho antes mencionado.

Estos hechos fueron denunciados, sin embargo no se obtuvieron las acciones legales equivalentes al daño ambiental generado, en razón a que los procedimientos administrativos originados, no son suficientes para sancionar el 
daño generado por la contaminación ambiental, siendo necesaria la utilización de otros mecanismos más efectivos para una adecuada protección del derecho fundamental al medio ambiente.

Según el numeral 2 del artículo 142 de la Ley General del Ambiente: "Se denomina daño ambiental a todo menoscabo material que sufre el ambiente y/o alguno de sus componentes, que pueda ser causado contraviniendo o no disposiciones jurídicas, y que genera efectos negativos actuales o potenciales", dichos efectos son externalidades que se transmiten a la sociedad o a las víctimas producto de la contaminación ambiental. Por lo que, las afectaciones medioambientales ostentan características especiales que las diferencian de los daños patrimoniales personales y que se resisten a ser atendidas por las disposiciones de derecho común referentes a la responsabilidad civil. "Los daños causados al medio ambiente inciden, como vimos en líneas anteriores, en dos ámbitos de alteración: el del patrimonio particular de un sujeto y en un bien de interés colectivo" (LÓPEZ SELA 2006, 309).

Actualmente, en razón a las diversas problemáticas generadas a causa de la vulneración del derecho fundamental al medio ambiente, el Poder Judicial y el Tribunal Constitucional son cuestionados por su labor, en razón a que las personas que recurren a estas instancias, a efectos de las diversas controversias sociales, no están satisfechas con la su labor, puesto que consideran que los fallos emitidos no protegen sus derechos, sin embargo es necesario señalar que muchas veces los afectados recurren a las vías ordinarias como son la penal, civil y administrativa, las mismas que demoran mucho, son costosas y no están configuradas para defender los derechos constitucionales, como si lo están los procesos constitucionales, que están diseñados para proteger los derechos fundamentales, garantizar la supremacía normativa de la Constitución y de toda fuente de derecho de rango constitucional.

Ahora, si consideramos que el derecho fundamental al medio ambiente de los pueblos indígenas es el que se vulnera en mayor medida a causa de las actividades extractivas, debemos considerar que este derecho tiene doble naturaleza, una de carácter individual y otra de carácter colectivo, motivo por el cual en la actualidad más litigantes recurren a la vía constitucional para proteger y garantizar dicho derecho, ello a través del ahora conocido Proceso de Amparo Ambiental. Prueba de ello es que se tiene diversos procesos sobre este tema, ya sea en primera y segunda instancia y también en el Tribunal Constitucional.

El proceso de amparo constituye el mecanismo ideal para la defensa del derecho fundamental al medio ambiente, mediante el cual se busca que "ante la negativa del poder político de cumplir con su obligación de proteger los derechos de los pueblos indígenas, la estrategia será exigir a los jueces que, a su vez le exijan al poder político, cumpla con su deber de garantizar los derechos de los pueblos indígenas" (RUIZ MOLLEDA 2013, 7). En los últimos años el Tribunal Constitucional dejó de ser 
un legislador negativo, que sólo tenía como función expulsar del ordenamiento jurídico a las normas que contradecían a la Constitución, para convertirse en la actualidad en un legislador positivo, es decir, creador de Derecho. Por lo tanto, a través de las sentencias emitidas por el Tribunal Constitucional, se pueden construir políticas públicas a favor del derecho al medio ambiente y de los derechos de los pueblos. Sin embargo, es necesario indicar, que el proceso de amparo en la actualidad tiene algunas falencias, que son atribuibles tanto a los operadores jurídicos, como a los litigantes, las cuales se deben superar para garantizar la defensa de nuestros derechos.

\section{Conclusiones}

Hasta ahora, se han dado políticas del Estado a favor de las empresas extractivas, que afectan de forma irreparable el medio ambiente y atentan contra los derechos de los pueblos indígenas, a través de la explotación indiscriminada de los recursos naturales.

Pese a que la Ley de consulta previa, buscaba evitar que se disponga de manera arbitraria de los territorios de los pueblos indígenas, en la realidad, constituye una mera formalidad que el Estado y las empresas extractivas deben cumplir, ya que es un mecanismo restringido y se realiza en condiciones de asimetría de poder, lo que ha generado que los conflictos sociales se sigan produciendo.

Ante esta desprotección por parte del Estado, la Constitución Política, nos da la posibilidad de defender los derechos antes mencionados, a través de las garantíass constitucionales, en particular en proceso de amparo. Asimismo, para evitar estos conflictos sociales, el Estado debe implementar medidas que armonicen la política energética y extractiva con los derechos fundamentales de los pueblos indígenas y la protección del medio ambiente, para que los intereses públicos no justifiquen la vulneración de los pueblos indígenas.

\section{Bibliografía}

ALAYZA MONCLOA, Alejandra. No pero sí: comunidades y minería: consulta y consentimiento previo, libre e informado. Lima: Cooper Acción, 2007.

COORDINADORA NACIONAL DE DERECHOS HUMANOS. Vulneraciones a los derechos de los pueblos indígenas en el Perú. Lima: Nauttica Media Design SAC, 2017.

DE ECHAVE CÁCERES, José. "Apurimac, la gestión del territorio y minería". Cusco: ditorial Grafica Bartolomé de las Casas S.A.C., 2012. 
DEFENSORIA DEL PUEBLO. Reporte de conflictos sociales No. 166. 15 de Diciembre de 2017 de 2017. http://www.defensoria.gob.pe/conflictos-sociales/.

ESCOBAR ROCA, Guillermo. Introducción a la Teoría Jurídica de los Derechos Humanos. Madrid: Trama, 2005.

LÓPEZ SELA, Pedro Luis y FERRO NEGRETE, Alejandro. “Derecho ambiental”. México: IURE Editores, 2006.

PALACÍN QUISPE, Miguel. "Impactos de la minería sobre los derechos de los pueblos indígenas". América Latina en movimiento. 15 de Marzo de 2017. www.américalatinaenmovimiento.com.pe (último acceso: 12 de Junio de 2018).

RUIZ MOLLEDA, Juan Carlos. “ ¿Por qué la consulta previa no funciona en proyectos extractivos en el Perú?" 25 de Marzo de 2015. http://www.redacción.lamu.pe (último acceso: 12 de 06 de 2018).

RUIZ MOLLEDA, Juan Carlos. Guía de litigio constitucional en defensa de los derechos de los pueblos indígenas para activistas de derechos humanos. Lima: Instituto de Defensa Legal, 2013.

TRIBUNAL CONSTITUCIONAL, Caso Alida Cortez Gómez de Nano. Sentencia 9642002-AA/TC (Tribunal Constitucional, 22 de 07 de 2002).

VIDAL RAMOS, Roger Pavletich. La Responsabilidad civil por daño ambiental en el sistema peruano. Lima: Editorial de la PUCP, 2013. 